\title{
Nonlinear Analysis of Rod Fastened Rotor under Nonuniform Contact Stiffness
}

\author{
Jiaqi Li $\mathbb{D}^{1,2}$ Yao Li $\mathbb{D},,^{1,2}$ Fan Zhang $\mathbb{D}^{1,2}$ and Yinli Feng $\mathbb{D}^{1,2}$ \\ ${ }^{1}$ Key Laboratory of Light Duty Gas Turbine, Institute of Engineering Thermophysics, Chinese Academy of Sciences, \\ Beijing 100190, China \\ ${ }^{2}$ University of Chinese Academy of Sciences, Beijing 100049, China
}

Correspondence should be addressed to Yinli Feng; fengyinli@iet.cn

Received 23 May 2020; Accepted 20 October 2020; Published 11 December 2020

Academic Editor: Lei Su

Copyright ( 2020 Jiaqi Li et al. This is an open access article distributed under the Creative Commons Attribution License, which permits unrestricted use, distribution, and reproduction in any medium, provided the original work is properly cited.

\begin{abstract}
Rod fastened rotor is widely used in gas turbine, aero engine, and other occasions. The bending stiffness of the contact interface directly affects the stable operation of the rotor. Dynamic model of the rod fastened rotor-bearing system has been established considering nonuniform stiffness of interface. The motion equation of this system has been deduced from Lagrange's equations. The linear dynamic characteristics of this rotor has been investigated, such as Campbell diagram, critical speed, and formation, and the nonlinear characteristics of this system, such as chaos and bifurcation, has been investigated too. The result shows that "bistable state" characteristic appeared on the rod fastened rotor system; that is, there are two critical speeds for each order, and they are all positive precession critical speed, and the amplitude response to the lower critical speed is much larger than that its counterparts to the higher critical speed. In terms of nonlinear characteristics, the rod fastened bearing system has experienced periodic 1 motion, multiple periodic motion, quasi-periodic motion, periodic 1 motion, and chaotic motion successively.
\end{abstract}

\section{Introduction}

The rotor-bearing system is the core component of rotating machines. Its dynamic characteristics affect the stable operation of rotary machinery directly. In order to prevent mechanical unstable operation, it is necessary to study the stability and nonlinear characteristics of rotor-bearing system. Studies on integral rotors are presented in literature [1-6]. Ehrich [1] studied the bifurcation phenomenon of Jeffcott rotor-bearing system and investigated the subharmonic vibration phenomenon in the rotor system. Wang [2] established the dynamic model of rub-impact rotor system and studied the periodic response stability of the system by Floquet theory. Gardner et al. [3] analyzed the nonlinear motion of the rotor system under the long bearing after linear instability and studied the subcritical and supercritical bifurcation in the method of multiscale. Bonello et al. [4] studied the nonlinear dynamic response of an extruded oil film damper-rotor system by harmonic balance method. Choi and Naoh [5] established the rotor model of bearing rubbing and analyzed the subharmonic, harmonic, and superharmonic vibration caused by rubbing. Yu et al. [6] studied the friction phenomenon of integral rotor by experimental method and discussed the influence of eccentricity, damping, and other factors on the nonlinear characteristics of this rotor.

The researches above focus on the integral rotor-bearing system. Rod fastened rotor has many advantages, such as high strength, light weight, and being easy to assemble and disassemble. The rod fastened rotor-bearing system is widely used in gas turbines and aeroengines (see Figure 1). Different from the integral rotor, the rod fastened rotor is not integral, and the disks at all levels are connected by the rod bolts. Therefore, its dynamic characteristics are quite different from the integral rotor. We have the following literature on the research of rod fastened rotor [7-12]. Hu et al. [7] considered the initial bending of the rotor when calculating the dynamics of the rod fastened rotor and systematically investigated the influence of rotational speed and initial bending on the dynamic response of the system. Hei et al. [8] 


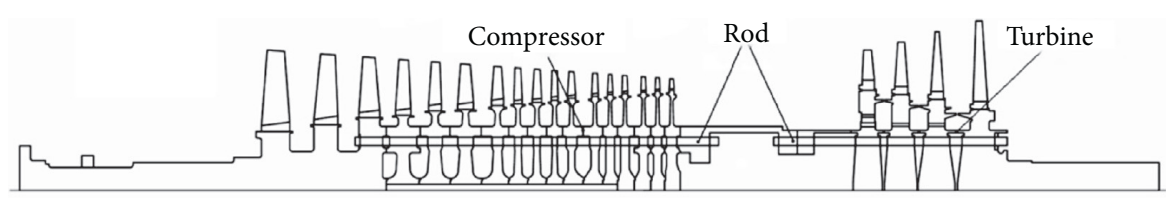

Figure 1: Rod fastened rotor model.

analyzed the dynamic characteristics of the fixed tilting pad bearing on the rotor-bearing system and studied the influence of the moment of inertia on the nonlinear characteristics of the system. Li et al. [9] built a model of rod fastened rotor by the three-dimensional finite element method and investigated the nonlinear dynamic characteristics of the rotor by Floquet method. Based on the harmonic balance method, the influence of system parameters on the nonlinearity of the rod fastened rotor was proposed by $\mathrm{Da}$ et al. [10]. Wang et al. [11] analyzed the influence of machining errors such as mass eccentricity on the nonlinear characteristics of rotor-bearing system of rod by the three-dimensional finite element method and Floquet theory. Yi et al. [12] proposed a calculation model considering mass effect for the flexible rotor-ball bearing system and analyzed the influence of preload and other factors on the nonlinear characteristics of the rotor subsystem.

The stiffness of the contact interface has a great influence on the dynamic characteristics of the rod fastened rotor. Unsuitable or nonuniform stiffness will generate system instability. The stiffness of the rod fastened rotor has been studied as follows [13-18]. Based on the theory of double friction, Liu et al. [13] analyzed the influence of preload force, gravity bending moment and other factors on the bending stiffness of the interface and verified this model by modal test. Isa et al. [14] calculated the bending stiffness under the action of gravity bending moment based on bilinear model and verified the simulation by experimental research. Lu et al. [15] calculated the natural frequencies under different preload by using the three-dimensional finite element model and analyzed the relationship between the natural frequencies and the preload. Gao et al. [16] simplified the bending stiffness to the stiffness of bending and twisting spring and analyzed the influence of preload on the bending stiffness of contact interface. Based on Hertz contact theory and Greenwood model, Rao [17] established a contact stiffness model of the rod fastened rotor and verified the model with hammering test. Xia et al. [18] proposed a piecewise bilinear torsional deformation mode and studied the influence of the preloading force and the friction coefficient of the contact interface on the bending stiffness of the contact interface.

In this paper, the dynamic characteristics of rod fastened rotor under nonuniform stiffness have been studied, such as Campbell diagram, critical speed, and formation. The dynamic model of rod fastened rotor-bearing system has been established. The fourth-order Runge-Kutta method is used to get the solution of the nonlinear model. Bifurcation diagram, frequency spectrum, phase trajectory, and Poincare map have been applied to study the nonlinear dynamic phenomena of the rod fastened rotor.

\section{Theoretical Analysis}

The rod fastened rotor is complicated; in order to simplify this model, the following assumptions are proposed:

(1) There is no torsional vibration and axial vibration in vibration process

(2) The disks at all levels do not separate, in vibration process

(3) Bearings at both sides of the rod fastened rotor are identical

2.1. Equation of Motion. As is shown in Figure 2, the rotors are closely connected through circumferential uniform rods, and the rotor is supported by sliding bearings.

$m_{b 1}$ and $m_{b 2}$ are the lumped mass of the rotor at the sliding bearing, respectively, while $m_{1}, m_{2}$, and $m_{3}$ are the lumped mass of the rotor at the interface, where $k$ is the stiffness of the shaft and $k_{2}$ and $k_{2}^{\prime}$ are the maximum bending stiffness and the minimum bending stiffness of interface of rod fastened rotor, respectively. $c_{1}$ is the bearing damping and $c_{2}$ is the rotor damping. According to the literature [7], the directions of $k_{2}$ and $k_{2}^{\prime}$ are perpendicular to each other, so the coordinate system is established with the direction of $k_{2}$ as the $x$-axis and the direction of $k_{2}^{\prime}$ as the $y$-axis.

The equation of the rod fastened rotor-bearing system can be deduced by Lagrange equation. Lagrange's equation can be described by

$$
\frac{\mathrm{d}}{\mathrm{d} t}\left(\frac{\partial L}{\partial \dot{q}_{i}}\right)-\frac{\partial L}{\partial \dot{q}_{i}}+\frac{\partial D}{\partial \dot{q} i}=f_{i}, \quad(i=1,2, \ldots, n),
$$

where $L$ is Lagrange function and $L=V-U, V$ is the kinetic energy of the system, $U$ is the potential energy of the system, $q_{i}$ and $q_{i}$ are the generalized coordinates and velocities of the system, $D$ is the dissipation energy of the system, and $f_{i}$ is the generalized force in the direction of $q_{i}$. The plane of the rotor center is zero potential energy reference surface of the gravitational potential energy.

The total kinetic energy of the rod fastened rotor system can be expressed as follows:

$$
\begin{aligned}
V= & \frac{1}{2} m_{b 1}\left(\dot{x}_{b 1}^{2}+\dot{y}_{b 1}^{2}\right)+\frac{1}{2} m_{1}\left(\dot{x}_{1}^{2}+\dot{y}_{1}^{2}\right)+\frac{1}{2} m_{2}\left(\dot{x}_{2}^{2}+\dot{y}_{2}^{2}\right) \\
& +\frac{1}{2} m_{3}\left(\dot{x}_{3}^{2}+\dot{y}_{3}^{2}\right)+\frac{1}{2} m_{b 2}\left(\dot{x}_{b 2}^{2}+\dot{y}_{b 2}^{2}\right) .
\end{aligned}
$$

The total potential energy of the rod fastened rotor system can be expressed as follows: 


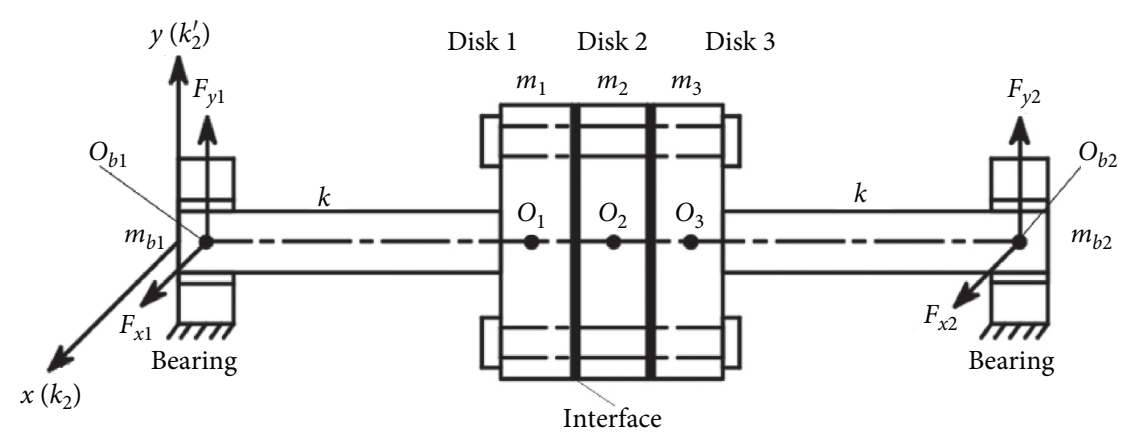

FIgURE 2: Dynamic model of a rod fastening rotor.

$$
\begin{aligned}
U= & \frac{1}{2} k\left(x_{b 1}-x_{1}\right)^{2}+\frac{1}{2} k\left(y_{b 1}-y_{1}\right)^{2}+\frac{1}{2} k_{2}\left(x_{1}-x_{2}\right)^{2}+\frac{1}{2} k_{2}^{\prime} y_{1}^{2}+\frac{1}{2} k_{2}\left(x_{2}-x_{3}\right)^{2} \\
& +\frac{1}{2} k_{2}^{\prime} y_{2}^{2}+\frac{1}{2} k_{2}\left(x_{3}-x_{b 2}\right)^{2}+\frac{1}{2} k_{2}^{\prime} y_{3}^{2}+\frac{1}{2} k\left(x_{b 2}^{2}+y_{b 2}^{2}\right) \\
& +m_{b 1} g y_{b 1}+m_{b 2} g y_{b 2}+m_{1} g y_{1}+m_{2} g y_{2}+m_{3} g y_{3} .
\end{aligned}
$$

The total dissipation energy of the rod fastened rotor system can be expressed as follows:

$$
\begin{aligned}
D= & \frac{1}{2} c_{1}\left(\dot{x}_{b 1}-\dot{x}_{1}\right)^{2}+\frac{1}{2} c_{1}\left(\dot{y}_{b 1}-\dot{y}_{1}\right)^{2}+\frac{1}{2} c_{2}\left(\dot{x}_{1}-\dot{x}_{2}\right)^{2}+\frac{1}{2} c_{2} \dot{y}_{1}^{2}+\frac{1}{2} c_{2}\left(\dot{x}_{2}-\dot{x}_{3}\right)^{2} \\
& +\frac{1}{2} c_{2} \dot{y}_{2}^{2}+\frac{1}{2} c_{2}\left(\dot{x}_{3}-\dot{x}_{b 2}\right)^{2}+\frac{1}{2} c_{2} \dot{y}_{3}^{2}+\frac{1}{2} c_{1}\left(\dot{x}_{b 2}^{2}+\dot{y}_{b 2}^{2}\right)
\end{aligned}
$$

where $x_{b i}, y_{\mathrm{bi}}$ present the displacement of $O_{b i},(i=1,2) . x_{i}, y_{i}$ present the displacement of $O_{i},(i=1,2,3)$.

In (3), $(1 / 2) k\left(x_{b 1}-x_{1}\right)^{2}+(1 / 2) k\left(y_{b 1}-x_{1}\right)^{2}$ represents the elastic potential energy of lumped mass of the rotor at the left sliding bearing, $(1 / 2) k_{2}\left(x_{1}-x_{2}\right)^{2}+(1 / 2) k_{2}^{\prime} y_{1}^{2}$ represents the elastic potential energy of $\operatorname{disk} 1,(1 / 2) k_{2}\left(x_{2}-x_{3}\right)^{2}+(1 / 2) k_{2}^{\prime} y_{2}^{2}$ represents the elastic potential energy of disk2, $(1 / 2) k_{2}\left(x_{3}-x_{b 2}\right)^{2}+(1 / 2) k_{2}^{\prime} y_{3}^{2}$ represents the elastic potential energy of disk3, and $(1 / 2) k\left(x_{b 2}^{2}+y_{b 2}^{2}\right)$ represents the dissipated energy the lumped mass of the rotor at the right sliding bearing.

In $(4), \quad(1 / 2) c_{1}\left(\dot{x}_{b 1}-\dot{x}_{1}\right)^{2}+(1 / 2) c_{1}\left(\dot{y}_{b 1}-\dot{y}_{1}\right)^{2}$ represents the dissipated energy of lumped mass of the rotor at the left sliding bearing, $(1 / 2) c_{2}\left(\dot{x}_{1}-\dot{x}_{2}\right)^{2}+(1 / 2) c_{2} \dot{y}_{1}^{2}$ represents the dissipated energy of disk1, $(1 / 2) c_{2}\left(\dot{x}_{2}-\dot{x}_{3}\right)^{2}+$ $(1 / 2) c_{2} \dot{y}_{2}^{2}$ represents the dissipated energy of disk2, $(1 / 2) c_{2}\left(\dot{x}_{3}-\dot{x}_{b 2}\right)^{2}+(1 / 2) c_{2} \dot{y}_{3}^{2}$ represents the dissipated energy of disk3, and $(1 / 2) c_{1}\left(\dot{x}_{b 2}^{2}+\dot{y}_{b 2}^{2}\right)$ represents the dissipated energy of the lumped mass of the rotor at the right sliding bearing.
The generalized coordinates of the system are $q_{i}=\left(x_{1}, y_{1}\right.$, $\left.x_{2}, y_{2}\right)^{\mathrm{T}}$, and submitting (2)-(4) into (1), the equation of motion can be deduced as follows:

$$
\left\{\begin{array}{l}
m_{1} \ddot{x}_{1}+\left(c_{1}+c_{2}\right) \dot{x}_{1}+\left(k_{2}-k\right) x_{1}=F_{x 1}, \\
m_{1} \ddot{y}_{1}+\left(c_{1}+c_{2}\right) \dot{y}_{1}+\left(k_{2}^{\prime}+k\right) y_{1}=F_{y 1}-m_{1} g, \\
m_{2} \ddot{x}_{2}+2 c_{2} \dot{x}_{1}+2 k_{2} x_{2}=m_{2} y_{2} \omega^{2} \cos (\omega t), \\
m_{2} \ddot{y}_{2}+2 c_{2} \dot{y}_{2}+k_{2}^{\prime} y_{2}=m_{2} y_{2} \omega^{2} \sin (\omega t)-m_{2} g, \\
m_{3} \ddot{x}_{3}+2 c_{2} \dot{x}_{3}+2 k_{2} x_{3}=F_{x 2}, \\
m_{3} \ddot{y}_{3}+c_{2} \dot{y}_{3}+k_{2}^{\prime} y_{3}=F_{y 2}-m_{3} g,
\end{array}\right.
$$

where, $F_{x 1}, F_{y 1}, F_{x 2}$, and $F_{y 2}$ are the components of the oil film forces on the left and right sides of the bearings in the $x$ and $y$-directions, respectively. Based on Assumption 2, it can be considered that $F_{x 1}=F_{x 2}, F_{y 1}=F_{y 2}$. 
2.2. Oil Film Force of Bearing. The sliding bearing model is shown in Figure 3, where $O_{b}$ is the bearing center, $O_{j}$ is the journal center, $\theta$ is the attitude angle, $F_{\varepsilon}$ is the radial component of the nonlinear oil film force on the axis diameter, and $F_{\theta}$ is the radial component of the nonlinear oil film force on the axis diameter. The oil film force of bearing can be obtained by solving the Reynolds equation.

Reynolds's equations are described by

$$
\frac{1}{R^{2}} \frac{\partial}{\partial \varphi}\left(\frac{h^{3}}{6} \frac{\partial p(\varphi, z)}{\partial \varphi}\right)+\frac{\partial}{\partial z}\left(\frac{h^{3}}{6} \frac{\partial p(\varphi, z)}{\partial z}\right)=\omega \frac{\partial h}{\partial \varphi}+2 \frac{\partial h}{\partial t}
$$

According to literature [19], when the oil film meets the following conditions, the oil is adiabatic, the flow of the oil is laminar, and the oil is incompressible; the oil film force can be expressed as follows:

$$
\left\{\begin{array}{l}
F_{\varepsilon}=\frac{6 \mu L R^{3} \omega}{c^{2}}\left\{\frac{2 \varepsilon^{2}(1-2 \dot{\theta})}{\left(2+\varepsilon^{2}\right)\left(1-\varepsilon^{2}\right)}+\frac{\left[\pi^{2}\left(2+\varepsilon^{2}\right)-16\right] \dot{\varepsilon}}{\pi\left(2+\varepsilon^{2}\right)\left(1-\varepsilon^{2}\right)^{1.5}}\right\}, \\
F_{\theta}=\frac{6 \mu L R^{3} \omega}{c^{2}}\left\{\frac{\pi \varepsilon(1-2 \dot{\theta})}{\left(2+\varepsilon^{2}\right)\left(1-\varepsilon^{2}\right)^{0.5}}+\frac{4 \dot{\varepsilon}}{\left(2+\varepsilon^{2}\right)\left(1-\varepsilon^{2}\right)}\right\},
\end{array}\right.
$$

where $\mu$ is kinematic viscosity of oil film, $L$ is the bearing length, $R$ is the bearing diameter, $\omega$ is angular velocity of bearing, $c$ is the bearing clearance, and $\varepsilon$ is the eccentricity ratio.

2.3. Bending Stiffness. Different from the integral rotor, rod fastened rotor can not be seen as a whole, the disks at all levels are connected by preload, and the bending stiffness of the contact interface plays an important role in the stability of the system. There are two methods to calculate bending stiffness: one is based on the theory of contact mechanics, the Green-Woods model, to calculate the bending stiffness, and another is calculated with finite element method. In this paper, the finite element method has been used to calculate the stiffness.

Figure 4 is a model of calculating the bending stiffness of a certain type of rod fastened rotor. The model consists of two disks and rods; there are four kinds of contact in this model: rough contact between the contact interface, no separation contact between the disk and rod, radial contact between two disks, plane contact between disk and rod nut, and boundary conditions and external load is shown in Figure 4. It should be noted that in order to adapt to the geometric characteristics of the disk, the rod nut has been modeled to be in the form of circle. Hexahedral elements have been used for mesh generation of this model. In order to improve the calculation accuracy, more precise grids have been arranged on the rod, as shown in Figure 5.

The stiffness of disks is

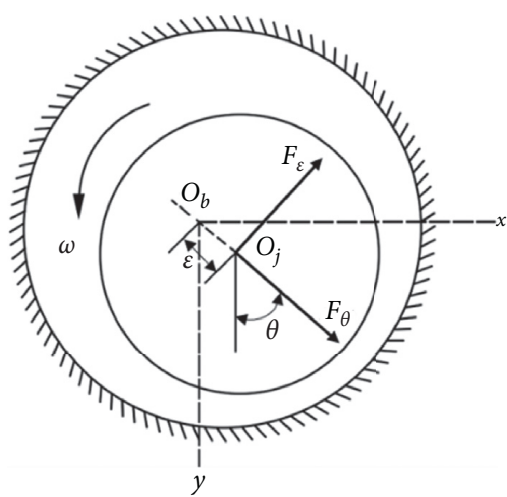

FIGURE 3: Model of sliding bearing.

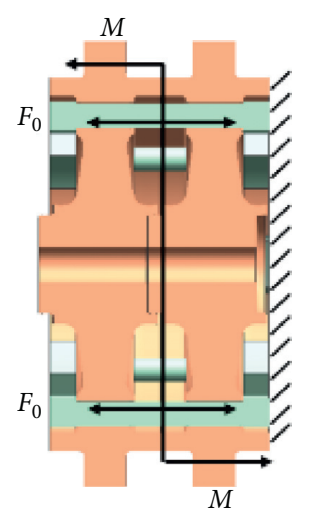

Figure 4: Model of bending stiffness.

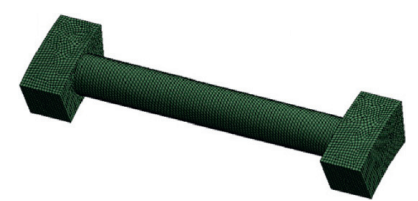

FIgURE 5: Mesh of the rod.

$$
K_{\mathrm{ra}}=\frac{M}{\alpha}
$$

The stiffness of rod is

$$
K_{\mathrm{rb}}=\frac{E A}{L_{1}} .
$$

The stiffness of rod fastened rotor is

$$
K_{r}=\frac{N K_{\mathrm{ra}} r_{p}^{2}}{2}+N K_{\mathrm{rb}}
$$

where $M$ is the bending moment applied and $\alpha$ is the deflection angle of the left disk, $E$ is the elastic modulus of the rod, $A$ is the cross-sectional area of the rod, $L_{1}$ is the length of the rod, $N$ is the number of rods, and $r_{p}$ is the radius at the center of the rod. 
2.4. Dimensionless Equation of Motion. In order to simplify the calculation, the dimensionless transformations are given as follows:

$$
\begin{aligned}
X_{i} & =\frac{x_{i}}{c}, \\
Y_{i} & =\frac{y_{i}}{c}, \\
\tau & =\omega t .
\end{aligned}
$$

According to (11), (5) can be simplified as

$$
\left\{\begin{array}{l}
m_{1} c \ddot{X}_{1}+\left(c_{1}+c_{2}\right) c \dot{X}_{1}+\left(k_{2}-k\right) c X_{1}=F_{x 10}, \\
m_{1} c \ddot{Y}_{1}+\left(c_{1}+c_{2}\right) c \dot{Y}_{1}+\left(k_{2}^{\prime}+k\right) c Y_{1}=F_{y 10}-m_{1} g, \\
m_{2} c \ddot{X}_{2}+2 c_{2} c \dot{X}_{2}+2 k_{2} c X_{2}=m_{2} c Y_{2} \omega^{2} \cos (\tau), \\
m_{2} c y \ddot{Y}_{2}+2 c_{2} c \dot{Y}_{2}+k_{2}^{\prime} c Y_{2}=m_{2} c Y_{2} \omega^{2} \sin (\tau)-m_{2} g \\
m_{3} c \ddot{X}_{3}+2 c_{2} c \dot{X}_{3}+2 k_{2} c X_{3}=F_{x 20}, \\
m_{3} c \ddot{Y}_{3}+c_{2} c \dot{Y}_{3}+k_{2}^{\prime} c Y_{3}=F_{y 20}-m_{3} g
\end{array}\right.
$$

where $F_{x 10}, F_{y 10}, F_{x 20}$, and $F_{y 20}$ are nondimensional oil film force of the bearing.

2.5. Solving Method. Considering the nonuniform bending stiffness of the rod fastened rotor, $k_{2} \neq k_{2}^{\prime}$, (12) has strong nonlinearity, and it is difficult to solve such problems by analytical method. Numerical integration methods are often used to solve the nonlinear problem.

Because of its high precision, Runge-Kutta method can calculate the influence of system parameters on system response, and it is the main method to solve nonlinear differential equations.

The normal form of the Runge-Kutta method is expressed as

$$
\left\{Y_{n+1}=Y_{n}+h \sum_{i=1}^{r} c_{i} K_{i}, \quad(i=1,2,3, \ldots, r), K_{1}=F\left(t_{n}, Y_{n}\right), K_{i}=F\left(t n+\lambda_{i} h, Y_{n}+h \sum_{j=1}^{i-1} \mu_{i j} K_{j}\right), \quad \lambda_{i}=\sum_{j=1}^{i-1} \mu_{i j}\right.
$$

After determining the order number, the coefficient $c_{i}, \lambda_{i}$, $\mu_{i j}$ can be determined through Taylor expansion and comparing coefficient.

The Fourth-order Runge-Kutta method is expressed as

$$
\left\{\begin{array}{l}
Y_{n}+1=Y_{n}+\frac{h}{6}\left[K_{1}+2 K_{2}+2 K_{3}+K_{4}\right] \\
K_{1}=F\left(t_{n}, Y\right) \\
K_{2}=F\left(t_{n}+\frac{1}{2} h, Y_{n}+\frac{h}{2} K_{1}\right) \\
K_{3}=F\left(t_{n}+\frac{1}{2} h, Y_{n}+\frac{h}{2} K_{2}\right) \\
K_{4}=F\left(t_{n}+h, Y_{n}+h K_{3}\right) .
\end{array}\right.
$$

\section{Results and Discussion}

The parameters of the rotor model have been shown in Table 1. Based on Assumption 2, the bearings on the left and right sides of the rotor are identical. Without considering nonuniform stiffness, the first- to third-order critical speed of this system is $470.63 \mathrm{rad} / \mathrm{s}, 1223.06 \mathrm{rad} / \mathrm{s}$, and $2022.56 \mathrm{rad} / \mathrm{s}$, respectively, as shown in Figure 6. The nonlinear dynamic behaviors of the rod fastened rotor-bearing system are performed by using the fourth-order Runge-Kutta method and implemented in MATLAB. Bifurcation diagram, time domain diagram, phase trajectory diagram, frequency spectrum, and Poincare map are used to illustrate the nonlinear characteristics.

The nonuniform bending stiffness will generate the circumferential anisotropy of the rod fastened rotor in the process of motion. In the analysis of the rotor fastened rotorbearing system, the bending stiffness is an important factor. Figure 6 shows the Campbell diagram of the rod fastened rotor under different degrees of nonuniform. It can be seen from Figure 6 that when the bending stiffness of the contact interface is nonuniform, the rotor appears to be "bistable state" phenomenon; that is, there are two critical speed of each order, and all of them are positive precession critical speed, and the higher critical speed is almost not affected by the nonuniform stiffness, while the smaller critical speed is greatly affected by $k_{2}^{\prime}$. Taking $k_{2}=5 k_{2}^{\prime}$ as an example, the firstto third-order formation of the rotor have been shown in Figure 7. As can be seen from Figure 7, the amplitude response to a smaller critical speed is much larger than its counterpart to a higher critical speed.

Figures $8-10$ are the bifurcation diagram of dimensionless horizontal displacement $\mathrm{X} 1$ at the right bearing location for three different types of stiffness $k_{2}=5 k_{2}^{\prime}$, $k_{2}=10 k_{2}^{\prime}, k_{2}=20 k_{2}^{\prime}$. It can be seen from Figure 8 that, at lower rotation speed, $\omega<2135 \mathrm{rad} / \mathrm{s}$, and the $\mathrm{rod}$ fastened rotor system maintains periodic 1 motion, which is shown as 
TABle 1: Parameters of the rod fastened rotor and bearing.

Parameters

Lumped mass $m_{1} / \mathrm{kg}$

Lumped mass $m_{2} / \mathrm{kg}$ Values

Lumped mass $m_{3} / \mathrm{kg}$

Lumped mass $m_{b 1} / \mathrm{kg}$

Lumped mass $m_{b 2} / \mathrm{kg}$

Stiffness of the shaft $k /(\mathrm{N} / \mathrm{m})$

Max stiffness of interface $k_{2} /(\mathrm{N} / \mathrm{m})$

Damp coefficient of bearing $c_{1}(\mathrm{~N} \cdot \mathrm{s} / \mathrm{m})$

Damp coefficient of rotor $c_{2}(\mathrm{~N} \cdot \mathrm{s} / \mathrm{m})$

Bearing radius $R(\mathrm{~mm})$

Bearing length $L_{b}(\mathrm{~mm})$

Clearance of bearing $c(\mathrm{~mm})$

Lubricant viscosity $\mu(\mathrm{Pa} \cdot \mathrm{s})$

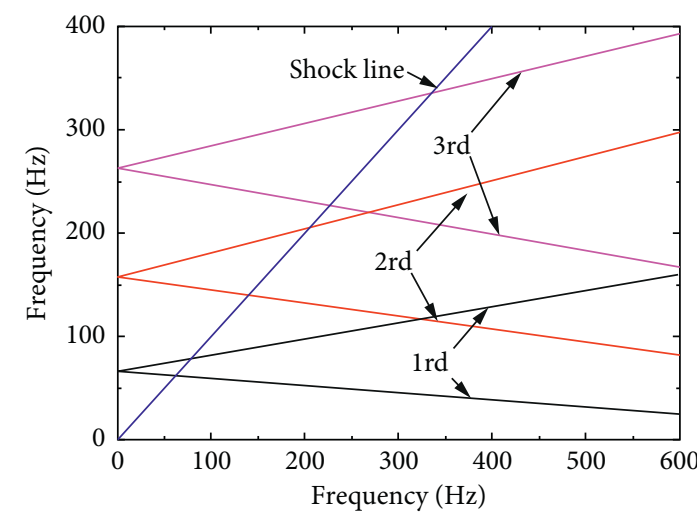

(a)

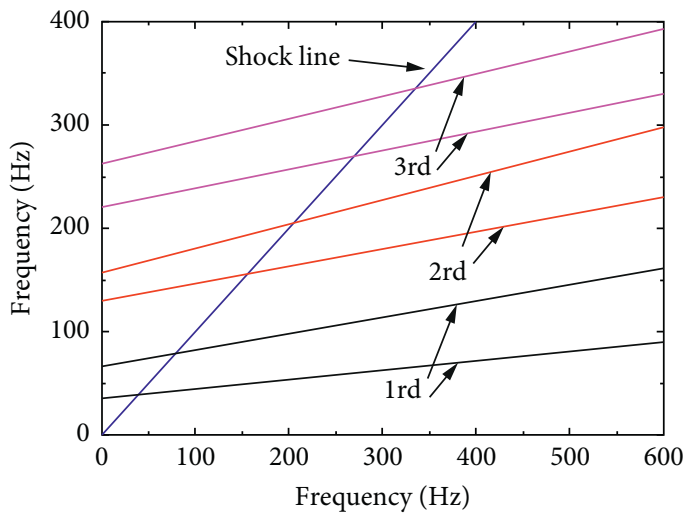

(c)

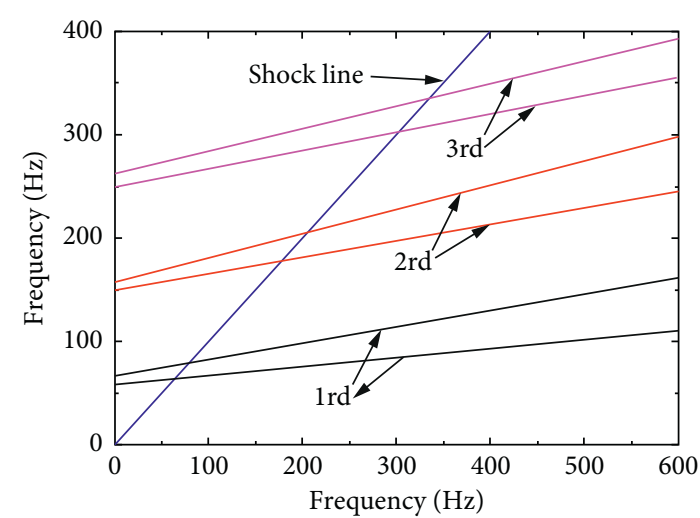

(b)

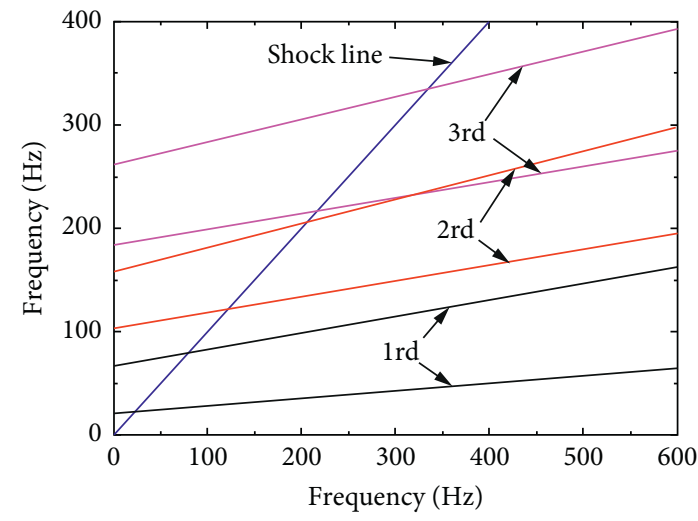

(d)

Figure 6: Campbell diagram of rod fastened rotor under nonuniform stiffness. (a) $k_{2}=k_{2}^{\prime}$, (b) $k_{2}=5 k_{2}^{\prime}$, (c) $k_{2}=10 k_{2}^{\prime}$, and (d) $k_{2}=20 k_{2}^{\prime}$.

a closed loop in Figure 11(b) and one isolated point in Figure 11(d). The system turns into a quasi-periodic motion, at a speed of $\omega=2135 \mathrm{rad} / \mathrm{s}$. As shown in Figure 12(b), the phase trajectory presents a lot of closed loops that do not overlap each other, and the Poincare map of the quasiperiodic motion presents a closed loop in Figure 12(d). Experienced a short quasi-periodic motion, $2135<\omega<2605 \mathrm{rad} / \mathrm{s}$, the system returns into periodic-1 motion again. With the increasing speed, $\omega>3745 \mathrm{rad} / \mathrm{s}$, the system response finally presents a chaos motion, which can be seen in Figure 13(d), Poincare map appears as messy, and irregular scatter.

Figure 9 is the bifurcation diagram of dimensionless horizontal displacement $X 1$ at the right bearing location for $k_{2}=10 k_{2}^{\prime}$. It can be seen from Figure 9 that the bifurcation diagrams of the system have a little difference. 

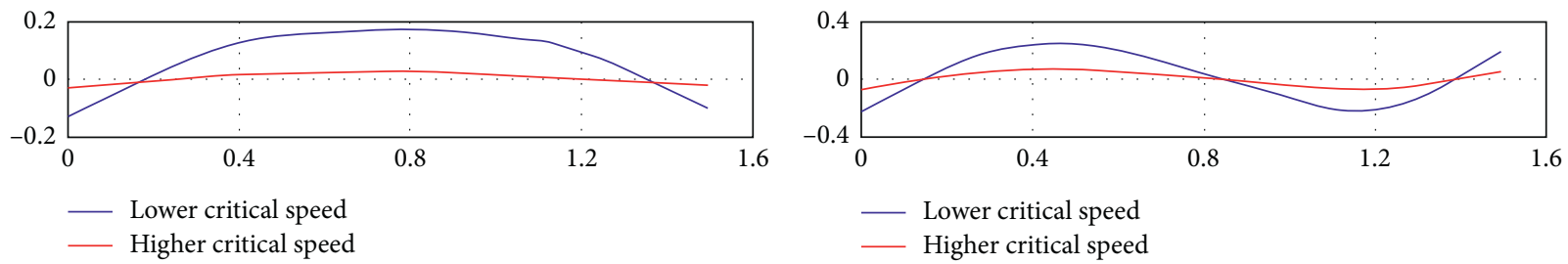

(a)

(b)

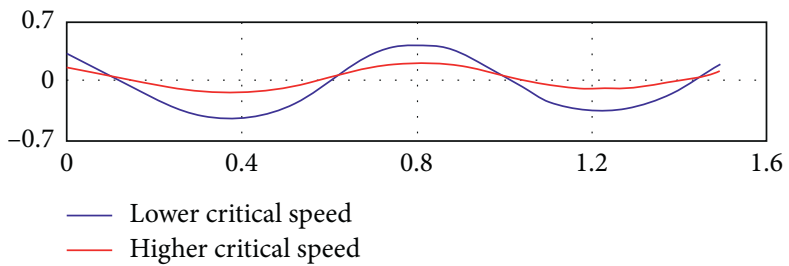

(c)

FIgURE 7: Mode shape of rod fastened rotor. (a) 1st mode shape, (b) 2nd mode shape, and (c) 3rd mode shape.

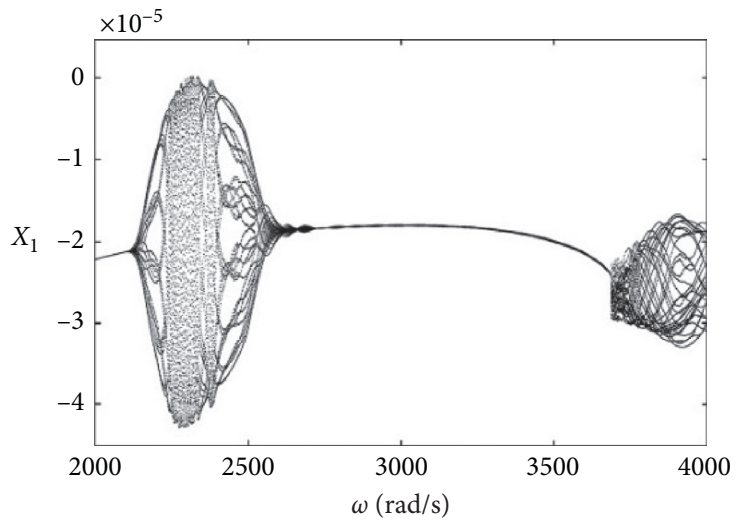

Figure 8: Bifurcation diagram of rotor with $k_{2}=5 k_{2}^{\prime}$.

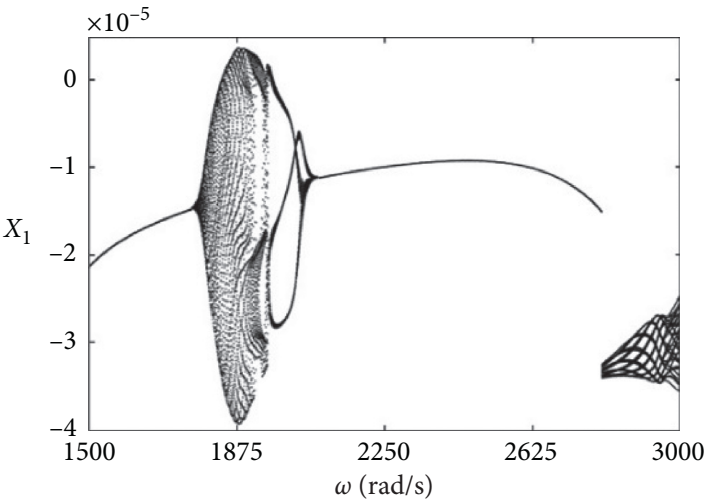

Figure 9: Bifurcation diagram of rotor with $k_{2}=10 k_{2}^{\prime}$.

System keeps period 1 motion, at low rotational speed, $\omega<1769 \mathrm{rad} / \mathrm{s}$. With the increasing of rotational speed, $1769<\omega<1987 \mathrm{rad} / \mathrm{s}$, system comes into quasi-periodic motion. $1875<\omega<1987 \mathrm{rad} / \mathrm{s}$; the system returns to periodic motion in the form of a period 3 inverse bifurcation; as shown in Figure 14(b), the phase trajectory presents three closed loops, and the Poincare map of the quasi-periodic motion presents three isolate points in Figure 14(d). With the increasing of speed, the system enters chaotic motion after a short period 1 motion.

Figure 10 is the bifurcation diagram of dimensionless horizontal displacement $X_{1}$ at the right bearing location 


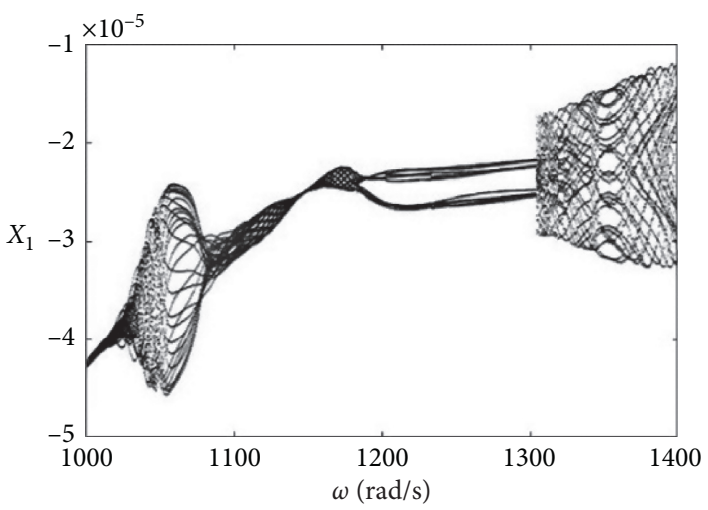

FIgURE 10: Bifurcation diagram of rotor with $k_{2}=20 k_{2}^{\prime}$.

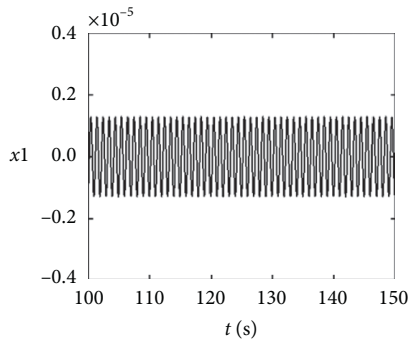

(a)

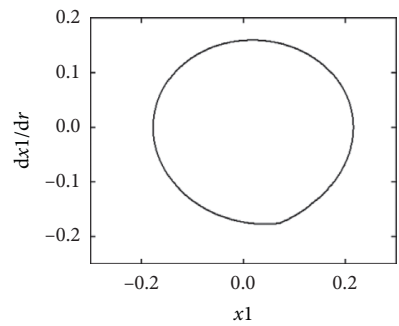

(b)

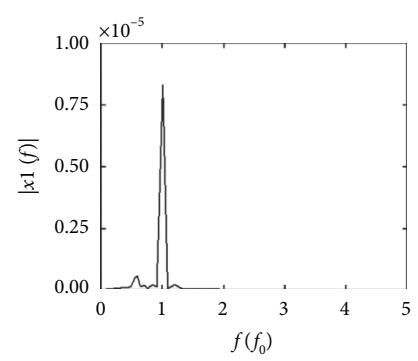

(c)

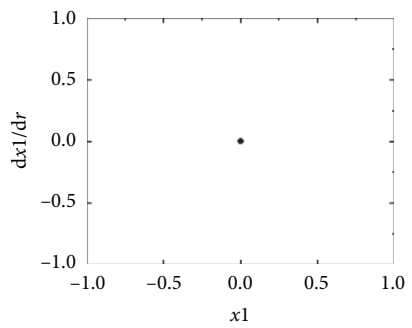

(d)

FIGURE 11: Numerical analysis results at $\omega=2000 \mathrm{rad} / \mathrm{s}, k_{2}=5 k_{2}^{\prime}$. (a) Time domain waveform, (b) phase trajectory, (c) frequency spectrum, and (d) Poincare map.

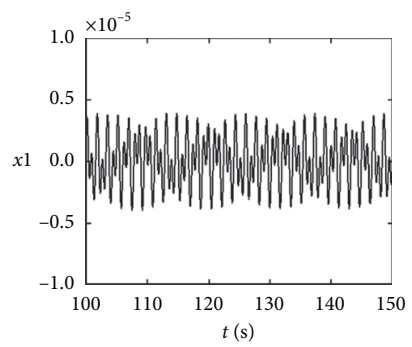

(a)

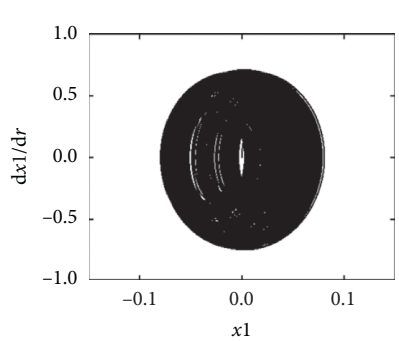

(b)

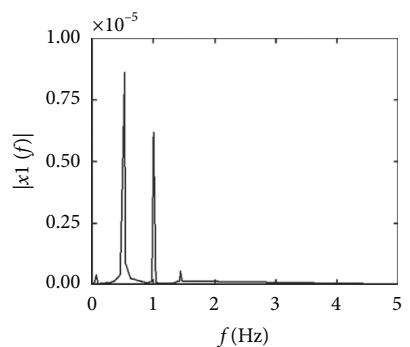

(c)

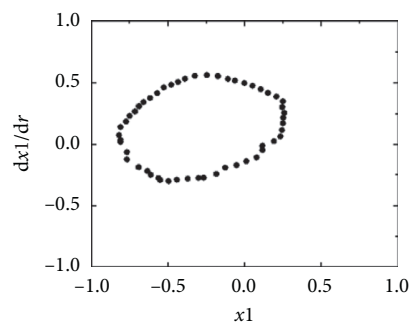

(d)

FIgURE 12: Numerical analysis results at $\omega=2605 \mathrm{rad} / \mathrm{s}, k_{2}=5 k_{2}^{\prime}$. (a) Time domain waveform, (b) phase trajectory, (c) frequency spectrum, and (d) Poincare map.

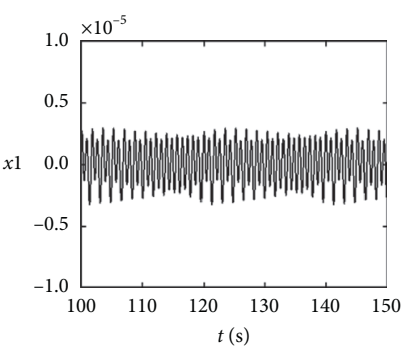

(a)

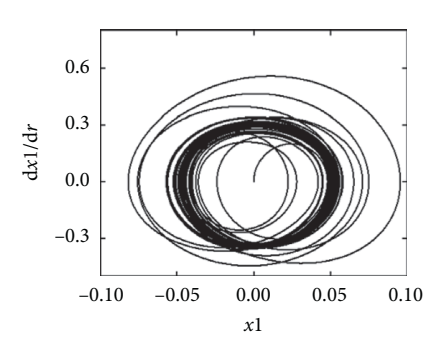

(b)

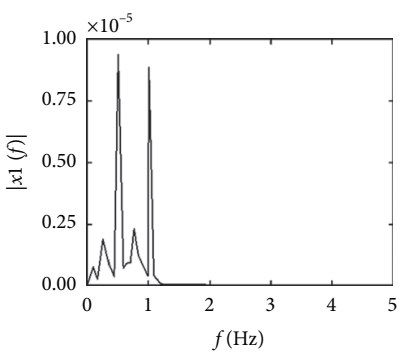

(c)

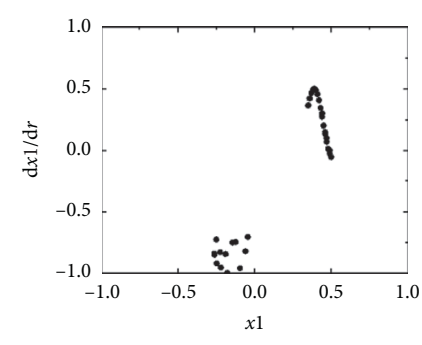

(d)

Figure 13: Numerical analysis results at $\omega=4000 \mathrm{rad} / \mathrm{s}, k_{2}=5 k_{2}^{\prime}$. (a) Time domain waveform, (b) phase trajectory, (c) frequency spectrum, and (d) Poincare map. 


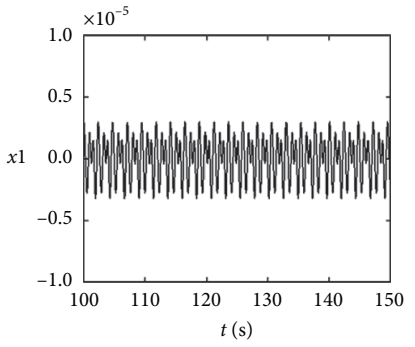

(a)

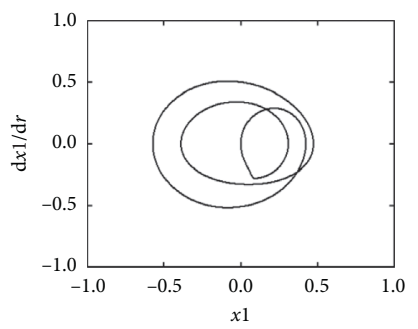

(b)

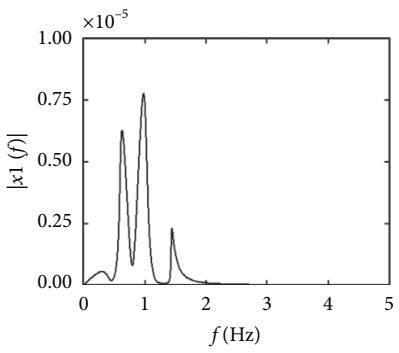

(c)

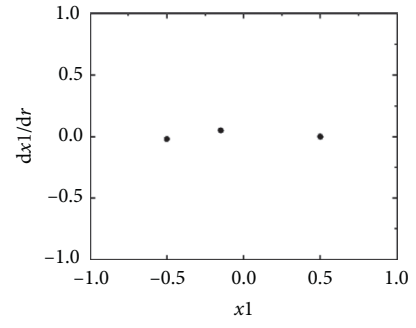

(d)

Figure 14: Numerical analysis results at $\omega=2061 \mathrm{rad} / \mathrm{s}, k_{2}=10 k_{2}^{\prime}$. (a) Time domain waveform, (b) phase trajectory, (c) frequency spectrum, and (d) Poincare map.

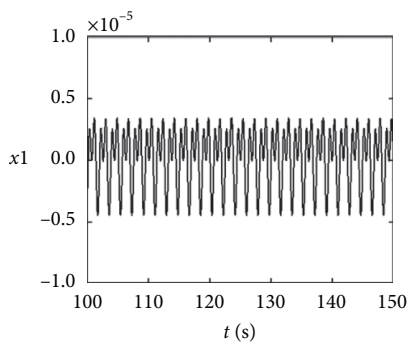

(a)

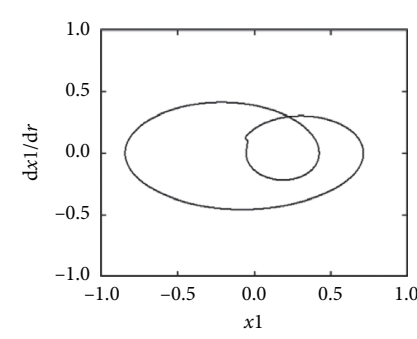

(b)

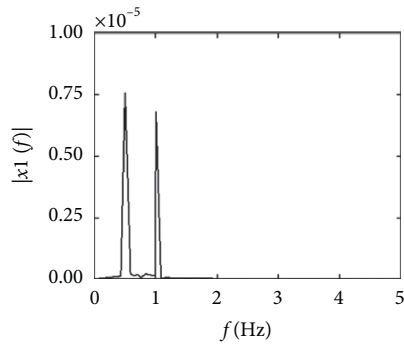

(c)

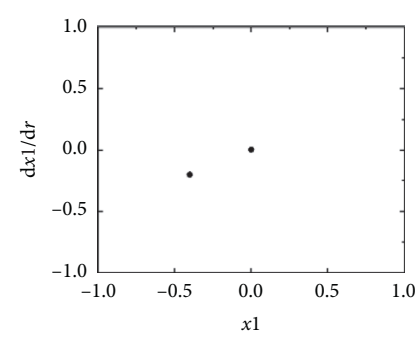

(d)

FIGURE 15: Numerical analysis results at $\omega=1250 \mathrm{rad} / \mathrm{s}, k_{2}=20 k_{2}^{\prime}$. (a) Time domain waveform, (b) phase trajectory, (c) frequency spectrum, and (d) Poincare map.

for $k_{2}=20 k_{2}^{\prime}$. The system response becomes more complicated under this condition. Compared with Figures 8 and 9, the quasi-periodic motion happens earlier and the quasi-periodic region narrows with $1027<\omega<1125 \mathrm{rad} /$ s. With the increase in the speed, system enters an unstable periodic motion, $1125<\omega<1193 \mathrm{rad} / \mathrm{s}$. At $\omega=1193 \mathrm{rad} / \mathrm{s}$, the system bifurcates again, and the system causes periodic 2 motion under certain conditions. Figure 13 is the response of the system at $\omega=1250 \mathrm{rad} / \mathrm{s}$, and the system presents periodic 2 motion, which is shown as two closed loops in Figure 15(b), two obvious frequency components in Figure 12(c), and two isolated points in Figure 15(d). As the rotational speed increases, the system enters chaotic motion without jump phenomenon.

\section{Conclusion}

Based on Lagrange's equation, the model of rod fastened rotor-bearing system under the condition of nonuniform bending stiffness of interface has been established in this paper. The linear dynamics behaviors have been explored, such as Campbell diagram and critical speed. The nonlinear dynamics behaviors have been studied by using the fourthorder Runge-Kutta method. The bifurcation diagram, vibration waveform, spectrum, phase trajectory, and Poincare map are given to illustrate the nonlinear dynamic phenomena of the system. The following conclusions can be drawn from the above research.
(1) With the increase of the rotating speed, the rod fastened rotor system has experienced periodic 1 motion, multiple periodic motion, and quasi-periodic motion successively. Then, the system returns to periodic 1 motion by an inverted bifurcation. With the speed increasing, the system enters chaotic motion in the end.

(2) With the increasing of $k_{2} / k_{2}^{\prime}$, the critical value of the system entering the chaotic phases is smaller, and the interval of quasi-periodic motion gets smaller.

(3) Because of the difference between $k_{2}$ and $k_{2}^{\prime}$, the rod fastened rotor system appears to be "bistable state" phenomenon. There are two critical speeds of each order, and both of them are positive precession critical speed. The smaller critical speed is greatly influenced by $k_{2}^{\prime}$, and the amplitude response to the smaller critical speed is much larger than the counterparts to the higher critical speed.

This study can provide guidance for the failure caused by nonuniform bending stiffness of the interface of the rod fastened rotor, and, at the same time, it is helpful to further study the nonlinear dynamic characteristics of the rod fastened rotor.

The further research work will focus on the modeling of the stability of the rod fastened rotor caused by the nonuniform stiffness. 


\section{Data Availability}

The data used to support the findings of this study are included within the article.

\section{Conflicts of Interest}

The authors declare that they have no conflicts of interest.

\section{Acknowledgments}

The associate professor Yang Jun of University of Shanghai for Science and Technology shows great concern for the author. Doctor Hu Liang of China University of Mining and Technology contributed greatly to this paper. Master Yan Tingfeng of East China University of Science and Technology contributed greatly to this paper. This work was supported by the National Science and Technology Major Projects (2017-IV-0010-0047).

\section{References}

[1] F. F. Ehrich, "Some observations of chaotic vibration phenomena in high-speed rotordynamics," Journal of Vibration and Acoustics, vol. 113, no. 1, pp. 50-57, 1991.

[2] J. Wang, J. Zhou, D. Dong, B. Yan, and C. Huang, "Nonlinear dynamic analysis of a rub-impact rotor supported by oil film bearings," Archive of Applied Mechanics, vol. 83, no. 3, pp. 413-430, 2013.

[3] M. Gardner, C. Myers, M. Savage, and C. Taylor, "Analysis of limit-cycle response in fluid-film journal bearings using the method of multiple scales," Quarterly Journal of Mechanics \& Applied Mathematics, vol. 38, no. 1, pp. 27-45, 1985.

[4] Bonello and Philip, The Non-Linear Modelling of Squeeze Film Damped Rotor-Dynamic Systems: An Efficient Integrated Approach, University of Southampton, Southampton, UK, 2002.

[5] S.-K. Choi and S. T. Noah, "Mode-locking and chaos in a Jeffcott rotor with bearing clearances," Journal of Applied Mechanics, vol. 61, no. 1, p. 131, 1994.

[6] J. J. Yu, P. Goldman, D. E. Bently et al., "Rotor seal experimental and analytical study on full annular rub," Journal of Engineering for Gas Turbine and Power, vol. 124, pp. 340-350, 2012.

[7] L. Hu, Y. Liu, L. Zhao, and C. Zhou, "Nonlinear dynamic behaviors of circumferential rod fastening rotor under unbalanced pre-tightening force," Archive of Applied Mechanics, vol. 86, no. 9, pp. 1621-1631, 2016.

[8] D. Hei, Y. Zhang, Z. Lu, P. Gupta, and N. Müller, "Nonlinear dynamic behaviors of a rod fastening rotor supported by fixed-tilting pad journal bearings," Chaos, Solitons \& Fractals, vol. 69, pp. 129-150, 2014.

[9] H. Lu, H. Liu, and L. Yu, "Nonlinear dynamic behaviors and stability of circumferential rod fastening rotor system," Journal of Mechanical Engineering, vol. 47, no. 23, pp. 82-91, 2011.

[10] Q. Da, Q. Yuan, and P. Li, "Nonlear dynamics of a rodfastened rotor system," Journal of Xian Jiaotong University, vol. 53, no. 5, pp. 43-51, 2019.

[11] Y. Liu, H. Liu, and N. Wang, "Effects of typical machining errors on the nonlinear dynamic characteristics of rod-fastened rotor bearing system," Journal of Vibration \& Acoustics
Transactions of the ASME, vol. 139, no. 1, Article ID 011004, 2017.

[12] J. Yi, H. Liu, Y. Liu, and M. Jing, "Global nonlinear dynamic characteristics of rod-fastening rotor supported by ball bearings," Proceedings of the Institution of Mechanical Engineers, Part K: Journal of Multi-Body Dynamics, vol. 229, no. 2, pp. 208-222, 2015.

[13] Y. Liu, Q. Yuan, P. Li, and G. Zhu, "Modal analysis for a rodfastened rotor considering contact effect based on double fractal model," Shock and Vibration, vol. 2019, Article ID 4027353, 10 pages, 2019.

[14] A. A. Isa, J. E. Mat, T. Penny, and S. D. Garvey, "The dynamics of bolted and laminated rotors," in Proceedings of SPIE-International Society for Optical Engineering, San Jose, CA, USA, February 2000.

[15] M. Lu, H. Geng, B. Yang, and L. Yu, "Finite element method for disc-rotor dynamic characteristics analysis of gas turbine rotor considering contact effects and rod preload," in Proceedings of the International Conference on Mechatronics \& Automation, Bangkok, Thailand, July 2010.

[16] J. Gao, Q. Yuan, P. Li et al., "Effects of bending moments and pretightening forces on the flexural stiffness of contact interfaces in rod-fastened rotors," Journal of Engineering for Gas Turbines and Power, vol. 134, no. 10, Article ID 102503, 2012.

[17] Z. Rao, "A study of dynamic characteristics and contacy stiffness of the rod fastening composite special rotor," Doctor thesis, Graduate Program in Engineering Science, Harbin Institute of Technology, Harbin, China, 1992.

[18] K. Xia, Y. Sun, D. Hong, J. Guo, and X. Kang, "Effects of contact interfaces on rotor dynamic characteristics of heavyduty gas turbine generator set," in Proceedings of the IEEE International Conference on Mechatronics \& Automation, Beijing, China, August 2020.

[19] M. Amira, "Nonlinear stability analysis of long hydrodynamic journal bearing using numerical continuation," Mechanism and Machine Theory, vol. 72, pp. 17-24, 2014. 